\title{
A Lower Bound for the Complexity of the Union-Split-Find Problem *
}

\author{
A $07 / 1986$ \\ Kurt Mehlhorn** \\ Stefan Näher** \\ Helmut Alt*** \\ **FB10, Informatik \\ Universität des Saarlandes \\ D-6600 Saarbrücken \\ Federal Republic of Germany \\ ***FB Mathematik, WE3 \\ Freie Universität Berlin \\ D-1000 Berlin 33 \\ Federal Republic of Germany
}

\begin{abstract}
We prove a $\Theta(\log \log n)$ (i.e. matching upper and lower) bound on the complexity of the Union-Split-Find problem, a variant of the Union-Find problem. Our lower bound holds for all pointer machine algorithms and does not require the separation assumption used in the lower bound arguments of Tarjan [T79] and Blum [B86]. We complement this with a $\Theta(\log n)$ bound for the Split-Find problem under the separation assumption. This shows that the separation assumption can imply an exponential loss in efficiency.
\end{abstract}

* This research was supported by the Deutsche Forschungsgemeinschaft, grant SPP Me $620 / 6-1$ 


\section{Introduction}

We consider the following three operations on a linear list $x_{1}, x_{2}, \ldots, x_{n}$ of items, some of which are marked.

$\operatorname{UNION}\left(x_{i}\right)$ : given a pointer to the marked item $x_{i}$ unmark this item

$\operatorname{SPLIT}\left(x_{i}\right)$ : given a pointer to the unmarked item $x_{i}$ mark this item

$\operatorname{FIND}\left(x_{i}\right):$ given a pointer to the item $x_{i}$ return a pointer to $x_{j}$ where $j=\min \left\{\ell \mid \ell \geq i\right.$ and $x_{\ell}$ is marked $\}$

Note that the marked items partition the linear list $x_{1}, \ldots, x_{n}$ into intervals of unmarked items. Then $\operatorname{FIND}\left(x_{i}\right)$ returns (a pointer to) the right endpoint of the interval containing $x_{i}, \operatorname{SPLIT}\left(x_{i}\right)$ splits the interval containing $x_{i}$, and $\operatorname{UNION}\left(x_{i}\right)$ joins the two intervals having $x_{i}$ as a common endpoint. We call the problem above the Union-Split-Find Problem; P.v.Emde Boas [EKZ77] called it a priority queue problem. He referred to the three operations as Insert, Delete and Successor, and exhibited a $O(\log \log n)$ solution for it. We will also consider the Split-Find Problem and the Union-Find Problem (only operations split, find and union, find respectively). Note that our Union-Find problem is a restriction of the usual Union-Find problem (here called the general Union-Find problem) because we allow only adjacent intervals to be joined. The Union-Split-Find problem is important for a number of applications, e.g. dynamic fractional cascading [MN86] and computing shortest paths [M84b, p.47].

We study the complexity of the Union-Split-Find problem in the pointer machine model of computation (Kolmogorov [Ko53], Knuth [Kn68], Schönhage [S73], Tarjan [T79]). A pointer machine captures the list processing capabilities of computers; its storage consists of records connected by pointers. Previously, lower bounds in a restricted pointer machine model (the term "restricted" is explained below) were obtained by Tarjan [T79] and Blum [B86]. Tarjan proved a $\boldsymbol{\Theta}(\alpha(n))$ bound on the amortized cost of the general Union-Find problem and Blum proved a $\Theta(\log n / \log \log n)$ bound on the worst-case cost. Tarjan's and Blum's lower bounds rely heavily on the following separation assumption (quoted from Tarjan [T79]):

"At any time during the computation, the contents of the memory can be partioned into collections of records such that each collection corresponds to a currently existing set, ... and no record in one collection contains a pointer to a record in another collection."

Because of this assumption we call their model restricted. If one views pointers as 
undirected edges in a graph then the separation assumption states that every currently existing set corresponds to a component of the graph.

The three main results of this paper are:

1. The complexity of the Union-Split-Find problem in the pointer machine model is $\boldsymbol{\Theta}(\log \log n)$. Here, the upper bound is on the worst-case cost of the three operations and the lower bound is on the amortized cost of the three operations, i.e., there are arbitrarily large $m$ and sequences of $m$ SPLIT, FIND and UNION operations having a total cost of $\Omega(m \log \log n)$.

The upper bound can be found in [EKZ77], [Ka84], and [MN86]. The solution of [MN86] supports two additional operations ADD and ERASE which allow to modify the underlying linear list; the solution does not satisfy the separation assumption.

The lower bound will be proved in section 2 of this paper.

2. In the restricted pointer machine model the worst-case complexity of the Split-Find problem is $\Theta(\log n)$ and the amortized complexity of the Union-Split-Find problem is $\boldsymbol{\Theta}(\log n)$.

This will be shown in section 3 .

3. a) (Tarjan [T79]).The amortized complexity of the Union-Find problem in the restricted pointer machine model is $\boldsymbol{\Theta}(\alpha(n))$.

b) Pointer machines obeying the separation assumption are exponentially weaker than pointer machines without the separation assumption.This is true for the worst-case complexity and for the amortized complexity

Tarjan proved his lower bound for the general Union-Find problem. His proof however is actually valid for the Union-Find problem considered here. b) follows immediately from results 1 and 2 . 


\section{The Lower Bound}

In this section we will show that each solution for the Union-Split-Find problem on a pointer machine requires $\Omega(\log \log n)$ computational steps, even in the amortized sense. More precisely, we show that there are arbitrarily large $m$ and sequences of $m$ UNION, FIND, SPLIT operations having a total cost of $\Omega(m \log \log n)$.

Our machine model is a pointer machine as described in [T79]. Its memory $M$ consists of an unbounded collection of records each containing 2 pointers to other records and an arbitrary amount of additional information. Thus $M$ can be regarded as a directed graph with outdegree 2 . We assume that the set of items $S$ is realized by two sets of records in $M$ a set of input records $I=\left\{x_{1}^{*}, x_{2}^{*}, \ldots, x_{n}^{*}\right\}$ and a set of output records $O=\left\{y_{1}^{*}, y_{2}^{*}, \ldots, y_{n}^{*}\right\}$. (The distinction between input and output records is merely a notational convenience.)

FIND $(x)$ is executed as follows. The machine starts with a pointer to input Record $x$ * (corresponding to item $x \in S$ ) in some register $r$. It stops with a pointer to the output record $y^{*}$ in $r$ which corresponds to item $y=\operatorname{FIND}(x)$. Since it can access records in $M$ only by pointers contained in one of its registers there must be a path $p$ formed by records and pointers in $M$ starting in $x^{*}$ and ending in $y^{*}$ such that pointers to all records on $p$ have been loaded into a register during the execution of the FIND. In addition to traversing a path from $x^{*}$ to $y^{*}$ the FIND operation may change some number of pointers. The cost of the FIND operation is certainly bounded from below by the length of a shortest path from $x^{*}$ to $y^{*}$ in $M$ plus the number of pointers changed.

When executing the operation $\operatorname{SPLIT}(y)$ the machine starts with a pointer to record $y^{*}$ in some register. After the operation record $y^{*}$ is reachable via pointers in $M$ for all records $x^{*}$ corresponding to items $x$ with $\operatorname{FIND}(x)=y$. To achieve this the machine changes certain pointers in $M$. The number of pointers changed is the cost of SPLIT $(y)$. The cost of UNION $(y)$ is defined similarly.

In order to model the actions of the pointer machine on its memory $M$ in a more abstract way we consider FIND and SPLIT as operations on a directed graph $G$ as follows:

Let $G=(V, E)$ be a directed graph with

1. For all $v \in V: \operatorname{outdegree}(v)=2$

2. There is a set of $n$ input nodes $I=\left\{x_{1}, x_{2}, \ldots, x_{n}\right\} \subseteq V$ 
3. There is a set of $n$ output nodes $O=\left\{y_{1}, y_{2}, \ldots, y_{n}\right\} \subseteq V-I$ which may be marked (we call $y_{i}$ the output node corresponding to input node $x_{i}, 1 \leq i \leq n$ ).

$\operatorname{FIND}\left(x_{i}\right), x_{i} \in I$, returns output node $y$, such that $j \geq i$ and $j$ is minimal with $y_{j}$ is marked. In addition, it may replace some edges of $G$ by new edges. The complexity of $\operatorname{FIND}\left(x_{i}\right)$ is the length of the shortest path from $x_{i}$ to $y_{j}$ in $G$ plus the number of edges replaced.

$\operatorname{SPLIT}(y), y \in O$,

marks output node $y$ and replaces some edges of $G$ by new edges such that every marked output node $y$ is reachable from all input nodes $x$ with $\operatorname{FIND}(x)=y$. The complexity of $\operatorname{SPLIT}(y)$ is the number of edges replaced.

$\operatorname{UNION}(y), y \in O$,

unmarks output node $y$ and replaces some edges of $G$ by new edges. The complexity of UNION $(y)$ is the number of edges replaced.

Lemma 1. Let $k$ be any integer, let $L=2^{2 k+1}+1$, let $n \geq 2^{(5 k) 2^{k}}$, and let $G_{0}=(V, E)$ be a directed graph with all output nodes unmarked. Then there is a sequence of $L$ SPLIT operations followed by $L$ FIND operations, followed by $L$ UNION operations such that

1. the total cost of the sequence is at least

$$
\min \left(k \cdot L, \frac{1}{2^{2 k+4}} n^{1 / 2^{k}}\right)
$$

2. all output nodes are unmarked after executing the sequence.

Proof. We call a graph $G$ a $\mathbf{k}$-structure iff for every input node $x$ there is a path of length at most $\mathrm{k}$ from $x$ to the output node $y=\operatorname{FIND}(x)$.

The idea of the proof is as follows. We first execute a sequence of $L$ SPLIT instructions (this sequence is constructed below) which leaves us with a data structure $G_{1}$. We next execute a hardest FIND on $G_{1}$, i.e. an operation $\operatorname{FIND}(x)$ where the shortest path from $x$ to $\operatorname{FIND}(x)$ has maximal length. Execution of this FIND instruction yields the data structure $G_{2}$. Again we perform a hardest FIND, . In this way we perform a total of $L$ FINDs, each FIND being a hardest FIND in the present data structure. Finally, we perform a sequence of UNIONs which undo all the SPLITs. This yields a data structure $G_{0}^{\prime}$ (generally different from $G_{0}$ ) in which all output nodes are unmarked.

In order to estimate the cost of this sequence of instructions we distinguish two cases. Assume first that no $G_{i}, 1 \leq i \leq L$, is a $k$-structure. Then each FIND costs at least $k$ 
time units for a total cost of $k \cdot L$ time units. Assume next that some $G_{i}$ is a $k$-structure. We will show that it takes at least $\left(1 / 2^{2 k+4}\right) \cdot n^{1 / 2^{k}}$ edge changes to construct $G_{i}$ from $G_{0}$; more precisely, we show the following claim.

Claim: Let $G_{0}$ be an arbitrary data structure with all output nodes unmarked. Then there is a set $\left\{z_{1}, \ldots, z_{L}\right\} \subseteq O$ of output nodes such that no $k$-structure $G^{\prime}$ with exactly $z_{1}, \ldots, z_{L}$ marked can be constructed from $G_{0}$ with less than $\left(1 / 2^{2 k+4}\right) \cdot n^{1 / 2^{k}}$ edge replacements.

Proof. For all $v \in V$ and $\ell \geq 1$ let $R_{\ell}(v)$ denote the set of all input nodes $w$ such that $v$ is reachable from $w$ on a path of length at most $\ell$.

Let $c_{0}, c_{1}, \ldots, c_{2^{k}}$ be a sequence of positive constants with

$$
n=c_{0} \geq c_{1} \geq \ldots \geq c_{2^{k-1}} \geq c_{2^{k}}=1 \text {. }
$$

Then the following lemma holds for $G_{0}$ :

Lemma 2. There is an $\ell, 0 \leq \ell \leq 2^{k}-1$, and $\ell$ distinct nodes $v_{1}, \ldots, v_{\ell}$ such that

1. either $\ell=0$ or $\left|\bigcap_{1 \leq i \leq \ell} R_{k-1}\left(v_{i}\right)\right| \geq c_{\ell}$

2. for each $w \in V-\left\{v_{1}, \ldots, v_{\ell}\right\}:\left|I^{*} \cap R_{k-1}(w)\right|<c_{\ell+1}$ where $I^{*}=\bigcap_{1 \leq i \leq \ell} R_{k-1}\left(v_{i}\right)\left(I^{*}=I\right.$ if $\left.\ell=0\right)$

Proof. Let $\ell_{0}$ be maximal such that there are distinct nodes $v_{1}, \ldots, v_{\ell_{0}}$ with $\mid \cap_{1 \leq i \leq \ell_{0}}$ $R_{k-1}\left(v_{t}\right) \mid>c_{\ell_{0}}$. We only have to show that $\ell_{0}<2^{k}$. Assume $\ell_{0} \geq 2^{k}$. Then there are $2^{k}$ distinct nodes $v_{1}, \ldots, v_{2^{k}}$ with $\cap_{1 \leq i \leq 2^{k}} R_{k-1}\left(v_{i}\right) \neq \emptyset$. But for every $x \in I$ there are at most $2^{k}-1$ nodes $v \in V$ with $x \in R_{k-1}(v)$, a contradiction.

Now let $\ell \geq 0$ and let $v_{1}, v_{2}, \ldots, v_{\ell}$ be nodes satisfying the conditions of lemma 2 . We give a sequence of $2^{2 k+1}+1$ SPLIT operations which require at least $\left(c_{\ell} / 2 L-2^{k+1}\right) / c_{\ell+1}$ edge replacements. First we divide $I^{*}=\cap_{1 \leq i \leq \ell} R_{k-1}\left(v_{i}\right)$ into $2 L$ about equal sized inlervals $A_{1}, A_{2}, \ldots, A_{2 L}$ i.e., $A_{1}$ consists of the $\left\lfloor I^{*} / 2 L\right\rfloor$ smallest indexed items in $I^{*}$, $A_{2}$ consists of the next $\left\lfloor I^{*} / 2 L\right\rfloor$ smallest indexed items in $I^{*}, \ldots$

Clearly,

$$
\left|A_{i}\right| \geq \frac{c_{\ell}}{2 L}-1 \text { for all } i .
$$

Let $A_{1}^{\prime}, A_{2}^{\prime}, \ldots, A_{2 L}^{\prime}$ be the corresponding intervals of output nodes.

Lemma 3. In every $A_{j}^{\prime}, j \in\{2,4,6, \ldots, 2 L\}$, there is an output node $y$, that is reachable in $G_{0}$ from at most $2^{k+1}$ input nodes in $A_{j-1}$ on a path of length $\leq k$. 
Proof. For all $x \in A_{j_{-1}}$ we have $\left|\left\{v \in V \mid x \in R_{k}(v)\right\}\right| \leq 2^{k+1}-1$. Thus we conclude

$$
\begin{aligned}
\sum_{x \in A_{j-1}}\left|\left\{v \in V \mid x \in R_{k}(v)\right\}\right| & \leq\left|A_{j-1}\right| \cdot\left(2^{k+1}-1\right) \\
& =\left|A_{j}^{\prime}\right| \cdot\left(2^{k+1}-1\right)
\end{aligned}
$$

Let $y_{j} \in A_{j}^{\prime}, j \in\{2,4, \ldots, 2 L\}$ be defined as in lemma 3 . Then there exists for each $j$ a set $B_{j-1} \subseteq A_{j-1}$ such that $\left|B_{j-1}\right| \geq\left|A_{j}\right|-2^{k+1}$ and such that for each $x \in B_{j-1}$ there is no path of length at most $k$ from $x$ to $y_{j}$ in $G_{0}$. Next consider any $k$-structure $G^{\prime}$ in which exactly the $y_{j}$ 's defined above are marked. In $G^{\prime}$ there must be a path of length at most $k$ from any $x \in B_{j-1}$ to $y_{j}$. Let $p(x)$ be any such path from $x$ to $y_{j}$ and let $e(x)$ denote the first new edge on $p(x)$. Let the edge $e(x)$ start in vertex $v(x)$. We have

\section{Lemma 4.}

1. $\left|\left\{x \in B_{j-1} \mid v(x)=w\right\}\right| \leq c_{\ell+1}$ for every node $w \notin\left\{v_{1}, v_{2}, \ldots, v_{\ell}\right\}$ and every $j$

2. There is a $j \in\{2,4,6, \ldots, 2 L\}$ such that $v(x) \notin\left\{v_{1}, v_{2}, \ldots, v_{\ell}\right\}$ for all $x \in B_{j-1}$.

\section{Proof.}

1. If $v(x)=w$ then $w$ was reachable from $x$ in the data structure $G_{0}$ in at most $k-1$ steps. Thus the claim follows immediately from the definition of $v_{1}, \ldots, v_{\ell}$.

2. For every $v \in\left\{v_{1}, v_{2}, \ldots, v_{\ell}\right\}$ we have $\left|\left\{y \in O \mid v \in R_{k}(y)\right\}\right| \leq 2^{k+1}$

and thus

$$
\sum_{v \in\left\{v_{1}, \ldots, v_{\ell}\right\}}\left|\left\{y \in O \mid v \in R_{k}(y)\right\}\right| \leq \ell 2^{k+1}
$$

$$
\leq 2^{2 k+1} \quad \text { since } \ell \leq 2^{k}
$$

i.e., all paths of length at most $k$ using nodes from $\left\{v_{1}, \ldots, v_{\ell}\right\}$ cannot lead to more than $2^{2 k+1}=L-1$ output nodes. Since we marked $L$ nodes in $O$ there must be one $y$, such that $v(x) \notin\left\{v_{1}, \ldots, v_{\ell}\right\}$ for all $x \in \boldsymbol{B}_{j-1}$.

Now consider any $j \in\{2,4, \ldots, 2 L\}$ satisfying condition 2 of lemma 4, i.e., $v(x) \notin$ $\left\{v_{1}, \ldots, v_{\ell}\right\}$ for all $x \in B_{j-1}$. Next observe that by part 1 of lemma 4 for any node $w \notin\left\{v_{1}, \ldots, v_{\ell}\right\}$ there are at most $c_{\ell+1}$ nodes $x \in B_{j-1}$ with $v(x)=w$. Thus

$$
\frac{\left|B_{j-1}\right|}{c_{\ell+1}}=\frac{\left|A_{j}\right|-2^{k+1}}{c_{\ell+1}}=\frac{\frac{c_{\ell}}{2 L}-1-2^{k+1}}{c_{\ell+1}} \geq \frac{\frac{c_{\ell}}{2^{2 k+2}+2}-1-2^{k+1}}{c_{\ell+1}}
$$


edges must be changed to obtain $G^{\prime}$ from $G_{0}$.

With $\quad c_{i}=n^{1-\frac{i}{2^{k}}}, \quad 0 \leq i \leq 2^{k}$

we conclude further that

$$
\begin{aligned}
\frac{\frac{c_{\ell}}{2^{2 k+2}+2}-\left(2^{k+1}+1\right)}{c_{\ell+1}} & \geq \frac{n^{\frac{1}{2^{k}}}}{2^{2 k+2}+2}-\left(2^{k+1}+1\right) \\
& \geq \frac{n^{\frac{1}{2^{k}}}}{2 \cdot\left(2^{2 k+2}+2\right)} \quad\left(\text { since } n \geq 2^{(5 k) 2^{k}}\right) \\
& \geq \frac{n^{\frac{1}{2^{k}}}}{2^{2 k+4}}
\end{aligned}
$$

This completes the proof of the claim and of lemma 1.

\section{Theorem 1.}

a) The single operation worst case complexity of the Split-Find problem is $\Omega(\log \log n)$.

b) The amortized complexity of the Union-Split-Find problem is $\Omega(\log \log n)$, i.e., there are arbitrarily large $m$ and sequences of $m$ UNION, SPLIT and FIND operations having a total cost of $\Omega(m \log \log n)$.

\section{Proof.}

a) Let $k$ be maximal such that $n \geq 2^{(5 k) 2^{k}}$. Then $k=\Omega(\log \log n)$ and

$$
\begin{aligned}
\frac{1}{2^{2 k+1}+1} \cdot \frac{1}{2^{2 k+4}} n^{\frac{1}{2^{k}}} & \geq \frac{2^{5 k}}{2^{2 k+4}\left(2^{2 k+1}+1\right)} \\
& =\Omega\left(2^{k}\right) \\
& =\Omega(\log n) \\
& =\Omega(\log \log n)
\end{aligned}
$$

Let $L=2^{2 k+1}+1$. Consider the sequence of $2 L$ SPLITs and FINDs constructed in the proof of lemma 1. The cost of this sequence is

$$
\Omega\left(\min \left(k \cdot L, \frac{1}{2^{2 k+4}} \cdot n^{1 / 2^{k}}\right)\right)=\Omega(L \cdot \log \log n)
$$


and hence at least one operation of the sequence has $\operatorname{cost} \Omega(\log \log n)$.

b) Let $k$ be maximal such that $n \geq 2^{(5 k) 2^{k}}$. Let $L=2^{2 k+1}+1$ and let $m=3 \cdot L \cdot s$ for some $s$. Let $G_{0}$ be any data structure with all output nodes unmarked. By part a) and lemma 1 there is a sequence of $3 \cdot L$ instructions such that

1. the total cost of the sequence is $\Omega(L \log \log n)$

2. all output nodes are unmarked after executing the sequence

Thus again by part a) and lemma 1 there is another sequence of $3 \cdot L$ instructions ... . Using this argument $s$ times yields a sequence of $m$ instructions with total $\operatorname{cost} \Omega(m \log \log n)$.

\section{A Lower Bound in the Restricted Model}

In this section we prove a $\Omega(\log n)$ lower bound for the worst-case complexity of the Split-Find problem and a $\Omega(\log n)$ lower bound for the amortized complexity of the Union-Split-Find problem in the restricted pointer machine model. We consider pointer machine algorithms satisfying the following separation condition:

The memory can be partioned into subgraphs such that each subgraph corresponds exactly to a current interval. There exists no edge from a node in such a subgraph to a node outside the subgraph ([B86], [T79]).

Similarly as in the previous section we define a k-structure.

Definition . Let $G=(V, E)$ be a directed graph with input nodes $I=\left\{x_{1}, \ldots, x_{n}\right\}$ and output nodes $O=\left\{y_{1}, \ldots, y_{n}\right\}$ some of which may be marked. $G$ is called a k-structure iff for every $x \in I$ there is a path of length at most $k$ to $\operatorname{FIND}(x)$ and $G$ fulfills the separation condition, i.e. for every $x_{i}, x_{j} \in I$ with $\operatorname{FIND}\left(x_{i}\right) \neq \operatorname{FIND}\left(x_{j}\right)$ there is no $v \in V$ that is reachable from both $x_{i}$ and $x_{j}$. 
Lemma 5. Let $n \geq(4 k)^{k} / k$ !. In any k-structure with output nodes $y_{1}, \ldots, y_{n-1}$ unmarked and $y_{n}$ marked satisfying the separation condition there is a SPLIT operation requiring at least $k / 12 \cdot n^{1 / k}$ edge replacements.

Proof. Since $y_{n}$ is the only marked output node there is for each input node $x$ a path of length at most $k$ to $y_{n}$. Let $T$ be the subgraph of $G$ formed by all shortest paths from input nodes to $y_{n}$. Then $T$ is a tree with root $y_{n}$, leaves $\left\{x_{1}, \ldots, x_{n}\right\}$ and height at most $k$. For any node $v$ in $T$ let $I(v)$ denote the set of input nodes which are the leaves of the subtree rooted at $v$. At each internal node $v$ of $T$ the incoming edges can be ordered $\left(w_{1}, v\right),\left(w_{2}, v\right), \ldots,\left(w_{m}, v\right)$ such that the minimal index of any input node in $I\left(w_{i}\right)$ is smaller than the minimal index of any input node in $I\left(w_{j}\right)$ for all $1 \leq i<j \leq m$. (We call $w_{i}$ the $i$-th son of $v$.)

Now assume that $\operatorname{SPLIT}(x)$ is executed for some $x \in I$. Let

$$
p: \quad x=v_{0} \longrightarrow v_{1} \longrightarrow \cdots \longrightarrow v_{\ell}=y_{n}
$$

be the path from $x$ to the root $y_{n}$ in $T$. For any $v_{i}, 1 \leq i \leq \ell$, on this path let $w_{1}^{i}, \ldots, w_{j}^{i}=v_{i-1}, \ldots, w_{m}^{i}$ be the sons of $v_{i}$ in the order defined above. Before the split operation $v_{i}$ is reachable from all input nodes of both of the following sets

$$
\begin{aligned}
& L_{\mathbf{i}}=\left\{u \mid u \text { is the node with minimal index in } I\left(w_{k}^{i}\right), 1 \leq k \leq j-1\right\} \\
& R_{\mathbf{i}}=\left\{u \mid u \text { is the node with minimal index in } I\left(w_{k}^{i}\right), j+1 \leq k \leq m\right\}
\end{aligned}
$$

After the split $\operatorname{FIND}(a) \neq \operatorname{FIND}(b)$ for all $a \in L_{i}, b \in R_{i}$ and by the separation condition $v_{i}$ is not reachable from any node of at least one of the sets $L_{i}$ or $R_{i}$. Thus the execution of $\operatorname{SPLIT}(x)$ requires at least

$$
\min \left(\left|L_{i}\right|,\left|R_{i}\right|\right)=\min \left\{j-1, \operatorname{indegree}\left(v_{i}\right)-j \mid v_{i-1} \text { is the } j \text {-th son of } v_{i}\right\}
$$

edge replacements for each node $v_{i}, 1 \leq i \leq \ell$, on path $p$.

The total cost for $\operatorname{SPLIT}(x)$ is

$$
c(p)=\sum_{i=1}^{\ell} \min \left\{j-1, \text { indegree }\left(v_{i}\right)-j \mid v_{t-1} \text { is the } j \text { th son of } v_{i}\right\}
$$

We first prove a lower bound for $c(p)$.

Lemma 6. Let $T$ be any tree of height $k$ with $n$ leaves and root $r$. Then there is a leaf $x$ in $T$ such that the path $p$ from $x$ to $r$ has cost

$$
c(p) \geq \frac{k}{12} \cdot n^{1 / k}
$$




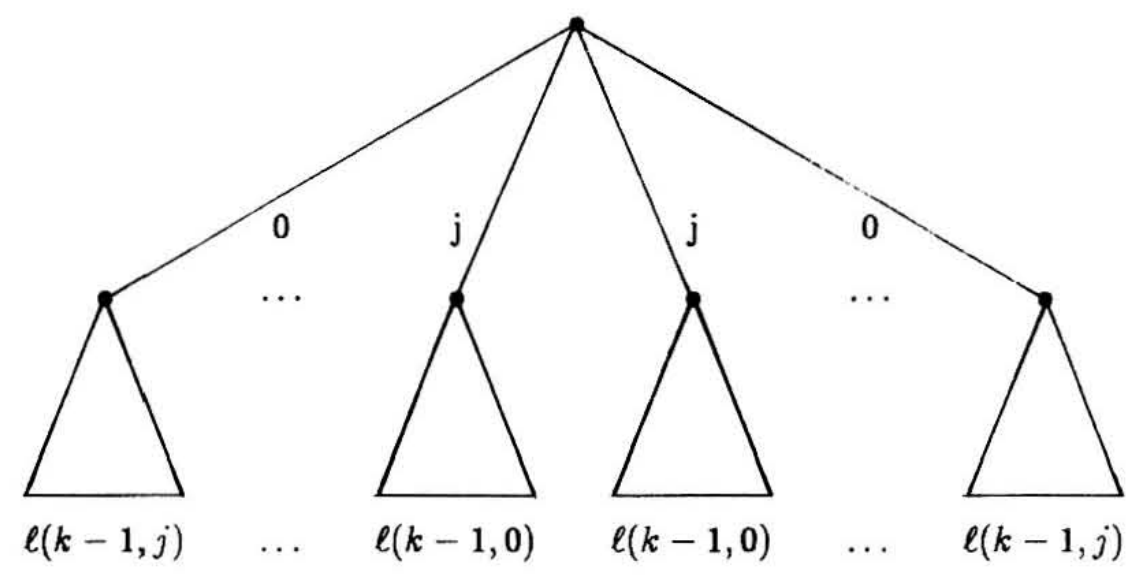

Figure 1

Proof. Define $\ell(k, j)$ as the maximal number of leaves in any tree $T$ of height $k$, such that $c(p) \leq j$ for every path $p$ from a leaf to the root of $T$

Then we have

$$
\ell(0, j)=1 \text { for } j \geq 0
$$

and (cf. figure 1)

$$
\begin{aligned}
\ell(k, j) & =2 \cdot \sum_{i=0}^{j} \ell(k-1, j-i) \\
& =2 \cdot \sum_{i=0}^{j} \ell(k-1, i) \text { for } k \geq 1
\end{aligned}
$$

The following table gives some values of $\ell(k, j)$. Figure 2 shows a tree of height $k=2$ with the maximal number of leaves such that all paths have at most cost $j=2$. 


\begin{tabular}{c|rrrrrrrrrr}
$\ell(k, j)$ & 0 & 1 & 2 & 3 & 4 & 5 & 6 & 7 & $\ldots$ & $k$ \\
\hline 0 & 1 & 2 & 4 & 8 & 16 & 32 & 64 & 128 & $\ldots$ & \\
1 & 1 & 4 & 12 & 32 & 80 & 192 & 448 & $\ldots$ & & \\
2 & 1 & 6 & 24 & 80 & 240 & 672 & $\ldots$ & & \\
3 & 1 & 8 & 40 & 160 & 560 & $\ldots$ & & & \\
4 & 1 & 10 & 60 & 280 & $\ldots$ & & & & \\
5 & 1 & 12 & 84 & $\ldots$ & & & & & \\
6 & 1 & 14 & $\ldots$ & & & & & & \\
7 & 1 & $\ldots$ & & & & & & & \\
$\vdots$ & & & & & & & & & \\
$j$ & & & & & & & & &
\end{tabular}

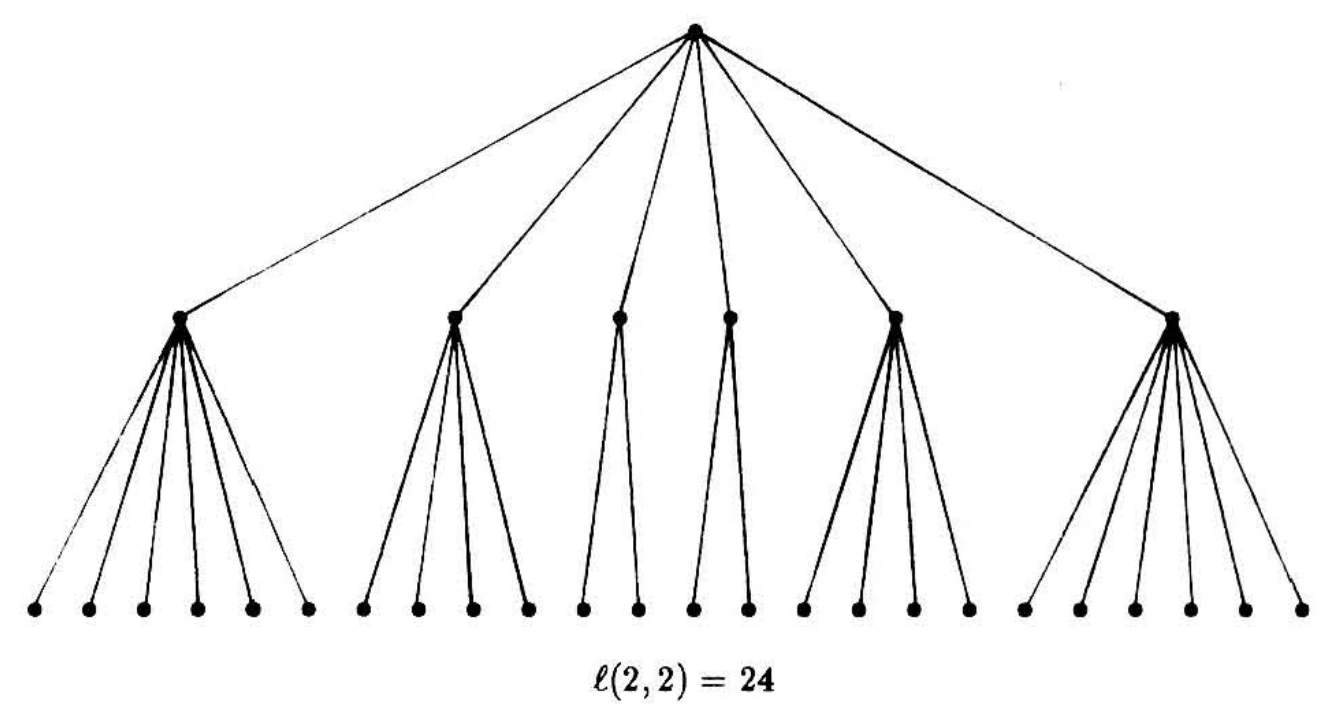

Figure 2

A different interpretation of $\ell(k, j)$ is as follows. Associate with every edge $e=(v, w)$ of $T$ the label $c(e)=\min \{i-1$, indegree $(w)-i \mid v$ is the $i$-th son of $w\}$. Then we have $0 \leq c(\boldsymbol{e}) \leq j$ for every edge $\boldsymbol{e}$ and for every path $\boldsymbol{p}=v_{0} \longrightarrow v_{1} \longrightarrow \cdots \longrightarrow v_{\boldsymbol{k}}$

$$
c(p)=\sum_{i=0}^{k-1} c\left(v_{i}, v_{i+1}\right)
$$

Thus $\quad \ell(k, j) \leq 2^{k}$. (number of possible ways to write $j$ as the sum of 
The term $2^{k}$ accounts for the fact that in every vertex each label can be used twice.

$$
\begin{aligned}
& \leq 2^{k} \cdot\left(\begin{array}{c}
k+j-1 \\
k-1
\end{array}\right) \\
& \leq 2^{k} \cdot \frac{(k+j-1)^{k-1}}{(k-1) !} \\
& \leq 2^{k} \cdot \frac{(k+j-1)^{k}}{k !} \\
& \leq 2^{k} \cdot \frac{(k+j)^{k}}{k !}
\end{aligned}
$$

We conclude that in any tree of height $k$ with $n$ leaves there exists a path $p$ from some leaf to the root such that

$$
\begin{aligned}
n & \leq 2^{k} \cdot \frac{(c(p)+k)^{k}}{k !} \\
& \leq 2^{k} \cdot \frac{(2 \cdot c(p))^{k}}{k !} \text { since } n \geq(4 k)^{k} / k ! \text { and hence } c(p) \geq k
\end{aligned}
$$

and finally

$$
\begin{aligned}
c(p) & \geq \frac{1}{2}\left(\frac{n \cdot k !}{2^{k}}\right)^{1 / k} \\
& \geq \frac{k}{12} \cdot n^{1 / k} \quad \text { since }(k !)^{1 / k} \geq \frac{k}{3} \text { if } k \geq 6
\end{aligned}
$$

This completes the proof of lemma 5 .

Theorem 2. In the restricted pointer machine model

a) the single-operation worst-case complexity of the Split-Find problem is $\Omega(\log n)$.

b) the amortized complexity for the Union-Split-Find problem is $\Omega(\log n)$, i.e. there are arbitrarily large $m$ and sequences of $m$ UNION, SPLIT, FIND operations having a total cost of $\Omega(\log n)$. 


\section{Proof.}

a) Let $k$ be maximal such that $n \geq(4 k)^{k} / k$ !. Then $k=\Omega(\log n)$. Let $G$ be any data structure with all output nodes except $y_{n}$ unmarked. By lemma 5 there is either a FIND operation which costs more than $k$ time units or there is a SPLIT operation having a cost of

$$
\frac{k}{12} \cdot n^{1 / k} \geq \frac{\log n}{12} \cdot n^{1 / \log n}=\frac{\log n}{6}=\Omega(\log n)
$$

b) Let $G$ be any structure with all output nodes except $y_{n}$ unmarked. Define $k$ as in part a). If $G$ is not a $k$-structure then we perform a hardest FIND in $G$; this FIND has cost $k=\Omega(\log n)$ and leaves us with a structure $G^{\prime}$ where all output nodes except $y_{n}$ are unmarked. If $G$ is a $k$-structure then there is a SPLIT operation of cost $k / 12 \cdot n^{1 / k}=\Omega(\log n)$. We perform it and immediately undo it by the corresponding UNION operation. This leaves us with a structure $G^{\prime}$ where all output nodes except $y_{n}$ are unmarked. At this point we are in the initial situation and we have forced the algorithm to spend $\Omega(\log n)$ time units on at most two operations. Part b) follows.

We want to point out that $O(\log n)$ is clearly also an upper bound for the complexity of the Union-Split-Find problem in the restricted model. One only has to represent each interval by a balanced tree; cf. e.g. [M84a, section III.5.3.1].

We close this section with our third result.

\section{Theorem 3.}

a) (Tarjan [T79]). The amortized complexity of the Union-Find problem in the restricted pointer machine model is $\Theta(\alpha(n))$.

b) Pointer machines obeying the separation assumption are exponentially weaker than pointer machines without the separation assumption.This is true for the worst-case complexity and for the amortized complexity

\section{Proof.}

a) Although Tarjan stated his result for the general Union-Find problem his proof actually works for our restricted version of it.

b) This follows immediately from theorem 1 and 2 . 


\section{Conclusions and Open Problems}

In this paper we proved several new lower bounds for the Union-Split-Find problem. In particular, we presented an $\Omega(\log \log n)$ lower bound for the amortized complexity of the Union-Split-Find problem valid for all pointer machine algorithms and an $\Omega(\log n)$ lower bound for restricted (in the sense of Tarjan [T79] and Blum [B86]) pointer machine algorithms. Our lower bounds match known upper bounds. Thus our results reveal that the separation assumption of Tarjan and Blum can imply an exponential loss in efficiency.

The following table summarizes all known bounds for the complexity of pointer machine algorithms for the Union-Split-Find problem on intervals including the results of this paper.

\begin{tabular}{|c|c|c|c|c|}
\hline Problem & \multicolumn{2}{|c|}{ General Model } & \multicolumn{2}{|c|}{ Restricted Model } \\
\hline $\begin{array}{l}\text { Union-Find } \\
\text { worst case } \\
\text { amortized }\end{array}$ & $\begin{array}{c}O(\log \log n) \\
-\end{array}$ & [EKZ77] & $\begin{array}{c}O(\log n / \log \log n) \\
\boldsymbol{\Theta}(\alpha(n))\end{array}$ & $\begin{array}{l}{[\mathrm{B} 86]} \\
{[\mathrm{T} 79]}\end{array}$ \\
\hline $\begin{array}{l}\text { Split-Find } \\
\text { worst case } \\
\text { amortized }\end{array}$ & $\begin{array}{c}\boldsymbol{\theta}(\log \log n) \\
-\end{array}$ & new & $\begin{array}{c}\boldsymbol{\theta}(\log n) \\
O\left(\log ^{*} n\right)\end{array}$ & $\begin{array}{c}\text { new } \\
{[\text { HU73] }}\end{array}$ \\
\hline $\begin{array}{l}\text { Union-Split-Find } \\
\text { worst case } \\
\text { amortized }\end{array}$ & $\begin{array}{l}\boldsymbol{\Theta}(\log \log n) \\
\boldsymbol{\Theta}(\log \log n)\end{array}$ & $\begin{array}{l}\text { new } \\
\text { new }\end{array}$ & $\begin{array}{l}\boldsymbol{\theta}(\log n) \\
\boldsymbol{\Theta}(\log n)\end{array}$ & $\begin{array}{l}\text { new } \\
\text { new }\end{array}$ \\
\hline
\end{tabular}

There are still several open problems in both models. We have no lower bounds for the amortized complexity of Union-Find and Split-Find and for the worst-case complexity of Union-Find in the general model. In the restricted model it remains an open problem whether the Split-Find algorithm of Hopcroft/Ullman [HU73] whose amortized running time is $O\left(\log ^{*} n\right)$ is optimal. We suppose that Blum's lower bound proof for the general Union-Find problem cannot be modified to work also for the interval problem.

We want to point out that the Union-Find problem and the Split-Find problem have amortized complexity $\Theta(1)$ on random access machines. This was shown by 
Garbow/Tarjan [GT83] for the Union-Find problem and by Imai/Asano [IA84] for the Split-Find problem.

\section{References}

[B86] N. Blum: "On the Single-Operation Worst-Case Time Complexity of the Disjoint Set Union Problem", SIAM J. Comput., Vol. 15, No. 4, 1986, 1021-1024

[EKZ77] P. v. Emde Boas, R. Kaas, E. Zijlstra: "Design and Implementation of an Efficient Priority Queue", Math. Systems Theory 10, 1977, 99-127

[GT83] H.N. Gabow, R.E. Tarjan: "A linear-time algorithm for a special case of disjoint set union", Proc. 15-th Ann. SIGACT Symp., 1983, 246-251

[IA84] T. Imai, T. Asano: "Dynamic Segment Intersection with Applications", 25th FOCS, 1984, 393-402

[HU73] J.E. Hopcroft, J.D. Ullman: "Set Merging Algorithms", SIAM J. Comput., Vol. 2, 1973, 294-304

[Ka84] R.G.Karlsson:" Algorithms in a Restricted Universe", Report CS-84-50, Department of Computer Science, University of Waterloo, 1984

[Kn68] D.E. Knuth: "The Art of Computer Programming", Vol. 3, "Fundamental Algorithms", Addison-Wesley, Reading, Mass., 1968.

[Ko53] A.N. Kolmogorov: "On the notion of Algorithm", Uspehi Mat. Nauk. 8 (1953), 175-176

[M84] K. Mehlhorn: "Data Structures and Algorithms", Springer Publ. Comp., 1984 
a) Vol. 1: Sorting and Searching

b) Vol. 2: Graph-Algorithms and NP-Completeness

c) Vol. 3: Multidimensional Searching and Computational Geometry

[MN86] K. Mehlhorn, S. Näher: "Dynamic Fractional Cascading", TR 06/1986, FB10, Universität des Saarlandes, Saarbrücken, 1986

[S73] A. Schönhage: "Storage Modification Machines", SIAM J. Comput., Vol. 9, 1980, 490-508

[T79] R.E. Tarjan: "A Class of Algorithms which Require Nonlinear Time to Maintain Disjoint Sets", J. Comp. Sys. Sci. 18, 1979, 110-127 